\title{
A High Flatness Gain Subsisting of Cascaded EDFA- TDFA Hybrid Optical Amplifier For Super Dense Wavelength Division Multiplexing System
}

Prof.Chakresh kumar ( $\nabla$ chakreshk.iit_dhanbad@rediffmail.com )

GGSIPU: Guru Gobind Singh Indraprastha University

Ghanendra Kumar

NIT DELHI

\section{Short Report}

Keywords: EDFA, TDFA, RAMAN, SD-WDM, gain, noise figure

Posted Date: July 15th, 2021

DOl: https://doi.org/10.21203/rs.3.rs-504312/v1

License: (c) (1) This work is licensed under a Creative Commons Attribution 4.0 International License.

Read Full License 


\section{Abstract}

Flat gain with least noise figure (NF) is the backbone feature of super dense wavelength division multiplexing (SD-WDM) system. It indicates the good amplification in terms of high-quality factor, lowest bit error rate, and good rating output power. But in this paper, we have mainly focused on characteristics of proposed EDFA-TDFA hybrid optical amplifier (HOA) in terms of flat gain and noise figure for C-Band. Evaluation has done for $400 \times 2.455 \mathrm{~Gb} / \mathrm{s}$ SD-WDM system with channel spacing of $0.8 \mathrm{~nm}$. Moreover, effect of proposed HOA also observed with displacement of channel wavelength for evaluating the impact of different set of HOA for analysis the same characteristics.

\section{Introduction}

Flat gain is the highly acceptable characteristics for super dense wavelength division multiplexing (SDWDM) system which try to archive with the combinations of different optical amplifiers and hybrid optical amplifiers (HOAs) [1-2]. Number of examples are given in the present literature such as combination of hybrid optical amplifiers and erbium-doped fiber amplifiers (EDFAs) in C plus $L$ band[3-4], combinations of thulium-doped fiber amplifiers (TDFAs) and (EDFAs) in $S$ plus $C$ plus $L$ band[5-6] and combination of (EDFAs) and Raman amplifiers (FRAs) in C plus $L$ plus $U$ band respectively[7]. But the combination of EDFA and TDFA is most significance hybrid optical amplifier (HOA) for delivering the flattered gain and also banished the effect of fiber nonlinearity [8-10]. Optical and at some place electrical pumping are the main biasing feature for the existing optical amplifier. Internal defects such as crosstalk and optical amplifier nonlinearity can also be control by adjusting the level of pumping power. Multiple pumping has also been recommended in the literature [11-13] for TDFA to enhance the performance of super multiplexing system in terms of bit error rate, output power, higher gain bandwidth, and noise figure. The performance of TDFA has also been observed for different cascaded amplification for different set of wavelength region [14-16].

In this paper to the best of authors knowledge, first time combination of EDFA and TDFA hybrid optical amplifier has been recommended for getting flat gain with insignificant effect of noise figure (NF) for super dense wavelength division multiplexing (SD-WDM) system.

However, paper is represented in IV sections. Section I, explained introduction of the paper, section II, explained the simulation setup of the proposed hybrid amplifier with dual pumping technique, section III, explained the final outcome from the proposed system and section IV, explained the final conclusion.

\section{Simulation Setup}

Our simulation setup for EDFA and TDFA hybrid amplifier is shown in Fig. 1.Simulation is carried out with dual pumping technique for both optical amplifiers. EDFA is pushed with dual pumping of $990 \mathrm{~nm}$ with $675 \mathrm{~mW}$ and $1490 \mathrm{~nm}$ with $540 \mathrm{~mW}$ respectively. In the similar manner, TDFA is pushed with dual pump of $1440 \mathrm{~nm}$ with $680 \mathrm{~mW}$ and $1460 \mathrm{~nm}$ with $690 \mathrm{~mW}$ respectively. 
In fact, carrier concentration in terms of population inversion can only be possible to set the level of pump laser diode (LD). Subsequently, outcome of the optical amplifier would be in good rating flattered gain with least noise figure [17].

Further, optical signal is fed to the EDFA amplifier then to cascaded TDFA amplifier. This is called EDFATDFA hybrid optical amplifier. The gain of EDFA is set to $25 \mathrm{~dB}$ with noise figure of $4 \mathrm{~dB}$. Optical signals are received at the receiver side by PIN photodiode, which is operated at $.875 \mathrm{~A} / \mathrm{W}$ of responsivity and 0.1 $\mathrm{nA}$ of dark current. Channel spacing of $0.8 \mathrm{~nm}$ is maintained for C-band.

In this proposed system, 400 optical signals are generated from $400 \mathrm{CW}$ laser sources. Power of each input probe is set to $-10 \mathrm{~mW}$ to maintain the quality of received signal [18]. Data rate is set to $2.455 \mathrm{~Gb} / \mathrm{s}$ NRZ binary sequence which further injected to sine-squared amplitude modulator to enhance the power level of low optical signal to higher optical signal level. Resultantly, the output is come out in terms of modulated signal.

\section{Result And Discussion}

Final outcome in terms of gain and noise figure with respect to C-band is shown in Fig. 2.The observed values for different sets of hybrid optical amplifiers in terms of flat gain are given as $20.1 \mathrm{~dB}$ to $18.55 \mathrm{~dB}$ for EDFA-TDFA HOA, 15.1dB-13.55dB for RAMAN-EDFA HOA, $10.1 \mathrm{~dB}$ to $8.55 \mathrm{~dB}$ for EDFA-EDFA HOA and $8.1 \mathrm{~dB}$ to $6.55 \mathrm{~dB}$ for RAMAN-RAMAN HOA respectively for the range of $1530 \mathrm{~nm}$ to $1565 \mathrm{~nm}$.

Recorded values of NFs are given as $8.5 \mathrm{~dB}$ for RAMAN-RAMAN HOA, 7.5 dB for EDFA-EDFA HOA, 7.2 dB for RAMAN-EDFA HOA and $2.5 \mathrm{~dB}$ for EDFA-TDFA respectively. It shows that our proposed HOA delivers the maximum gain of $25.55 \mathrm{~dB}$ with variation of $1.22 \mathrm{~dB}$ than the reference [17] which is good sign for optical communication.

However, performance of proposed hybrid amplifier is also evaluated with displacement in the channel wavelength in Fig. 3. It is clearly observed that the impact of proposed HOA still maintained in terms of flat gain with least variation of $1.5 \mathrm{~dB}$. But we can also notice that gain is linearly increasing till $1555 \mathrm{~nm}$ and decrease continuously till $1570 \mathrm{~nm}$ due to nonlinearity effect induced by hybrid optical amplifier. It is basically occurred due to the retardation of optical signal but it can also be improved by adjusting the power level of CW laser and power range of optical pump. Further, eye diagrams from different set of HOAs are also shown from Fig. 4 to Fig. 7 respectively to explore the impression of individual HOA. Nonlinear effect has highly dominating nature over optical communication which can only maintained with suitable rating of power amplification which has also done with the arrangement of EDFA-TDFA HOA. In fact, power strength of optical signals must be retained throughout the optical communication which further lead to good quality optical signals with least effect of dominating signals. The impression of proposed HOA is also observed in terms of power amplification in optical medium and at the receiver end which is also shown in Fig. 8 and Fig. 9 respectively. So, the eye opening of sudden checking quality of the transmission signals are also with the support of proposed HOA in Fig. 10. So, here it is observed 
that overall impression of EDFA-TDFA HOA is acceptable to maintain the flat gain with acceptable rating noise figure.

\section{Conclusion}

Performance of proposed EDFA-TDFA hybrid optical amplifier for $400 \times 2.455 \mathrm{~Gb} / \mathrm{s}$ super dense wavelength division multiplexing (SD-WDM) system has been done in terms of flat gain and noise figure. Maximum gain of $25.55 \mathrm{~dB}$ with least variation of $1.22 \mathrm{~dB}$ is recorded. Impact of HOA is really helped out to maintain the flat gain for the range of $1530 \mathrm{~nm}$ to $1570 \mathrm{~nm}$. On the other hand, influence of proposed hybrid optical amplifier ( $\mathrm{HOA}$ ) still retains with displacement of channel wavelength in terms of flat gain with variation of $1.5 \mathrm{~dB}$.

\section{Declarations}

Acknowledgment Authors are thankful to Guru Gobind Singh Indraprastha University and National Institute of Technology Delhi for providing the platform to conduct this research work.

Author Contributions Design and analysis a super dense optical communication to achieve the acceptable flat gain with the support of EDFA-TDFA Hybrid Optical Amplifier.

Funding The authors declare that they have no funding available to conduct this research work

Data Availability Data used for the results are available in the manuscript

Declarations Not applicable.

Conflict of Interest Authors declare that there is no conflict of Interest.

Consent to Participate We here give our consent to participate and communicate paper in this journal.

Consent for Publication We here give our consent to publish paper in this journal.

\section{References}


1- J. Kani, K. Hattori, M. Jinno, T. Kanamori, and K. Oguchi, "Triplewavelength-band WDM transmission over cascaded dispersion-shifted fiber," IEEE Photon. Technol. Lett., vol. 11, no. 11, pp. 1506-1508,Nov. 1999.

2- $\quad$ H. Masuda and S. Kawai, "Ultra wide-band Raman amplification with total gain-bandwidth of $132 \mathrm{~nm}$ of two gain-bands around $1.5 \mu \mathrm{m}$," in Proc. ECOC, 1999, vol. 2, pp. 146-147.

3- $\quad$ S. Aisawa, T. Sakamoto, F. Fukui, J. Kani, M. Jinno, and K. Oguchi,“Ultra-wide band long distance WDM transmission demonstration of $1 \mathrm{~Tb} / \mathrm{s}(50 \times 20 \mathrm{~Gb} / \mathrm{s}), 600 \mathrm{~km}$ transmission using 1550 and 1580 nm wavelength bands," Electron. Lett., vol. 34, no. 11, p. 1127, May 1998.

4- Y. Sun, J. W. Sulhoff, A. K. Srivastava, A. Abramov, T. A. Strasse,P. F. Strasse, P. F. Wyscocki, J. R. Pedrazzani, J. B. Judkins,R. P. Espindola, C. Wolf, J. L. Zyskind, A. M. Vengsarkar, and J. Zhou,"A gain-flattened ultra wide band EDFA for high capacity WDM optical communications systems," in Proc. Eur. Conf. Optical Communications,

Madrid, Spain, 1998, p. 53.

5- $\quad$ K. Fukuchi, T. Kasamatsu, M. Morie, R. Ohhira, T. Ito, K. Sekiya,D. Ogasahara, and T. Ono, "10.92$\mathrm{Tb} / \mathrm{s}$ (273/spl times/40-Gb/s) tripleband/ultra-dense WDM optical-repeatered transmission experiment," in Proc. Optical Fiber Communication (OFC), Anaheim, CA, 2001,pp. PD24-1-PD243.

6- J. Kani, K. Hattori, M. Jinno, S. Aisawa, T. Sakamoto, and K. Oguchi,“Trinal-wavelength-band WDM transmission over dispersion-shiftedfiber," in Proc. Optical Fiber Communication (OFC), San Diego, CA,1999, vol. 2, pp. 159-161.

7- $\quad$ T. Matsuda, T. Kotanigawa, and A. Naka, "90 × $42.7 \mathrm{~Gb} / \mathrm{s}(3.6 \mathrm{~Tb} / \mathrm{s})$ WDM signal transmission with triple band in-line amplifiers," presented at the Optical Amplifiers and Their Applications (OAA), San Francisco,CA, 2004, Paper OTuC3.

8- $\quad$ T. Sakamoto, A. Mori, and M. Shimizu, "Rare-earth-doped fiber amplifiers for eight-channel CWDM transmission systems," presented at the Optical Fiber Communication (OFC), Los Angeles, CA, 2004,Paper ThJ5.

9- T. Sakamoto, S. Aozasa, M. Yamada, and M. Shimizu, "High-gain hybrid amplifier consisting of cascaded fluoride-based TDFA and silica-basedEDFA in 1458-1540 nm wavelength region," Electron. Lett., vol. 39,no. 7, pp. 597-599, Apr. 2003.

10- T. Segi, T. Aizawa, T. Sakai, and A. Wada, "Silica-based composite fiber amplifier with 14801560 nm seamless gain-band," in Proc. Eur. Conf.Optical Communication (ECOC), Amsterdam, The Netherlands, 2001,pp. 228-229.

11- R. Singh, M.L. Singh, B. Kaur, A novel triple pump $1050 \mathrm{~nm}, 1400 \mathrm{~nm}, 800 \mathrm{~nm}$ pumping scheme for thulium doped fiber amplifier, Optik 123 (2012)1815-1816.

12- W.J. Lee, C.H. Lee, P. Kim, J.-O. Byun, J. Park, N. Park, Study on the gain excursion and tilt compensation for 1.4- and 1.5-m dual wavelength pumped TDFA, IEEE Photonics Technol. Lett. 14 (6 (June)) (2002) 786-788.

13- S.D. Emami, S.W. Harun, et al.,Optimization of the $1050 \mathrm{~nm}$ pump power and fiber length in single-pass and double-pass thulium doped fiber amplifiers, Prog.

Electromagn. Res. B 14 (2009) 431-448.

14- T. Sakamoto, A. Mori, and M. Shimizu, "Rare-earth-doped fiber amplifiers for eight-channel CWDM transmission systems," presented at the Optical Fiber Communication (OFC), Los Angeles, CA, 2004,Paper ThJ5. 
15- T. Sakamoto, S. Aozasa, M. Yamada, and M. Shimizu, "High-gain hybrid amplifier consisting of cascaded fluoride-based TDFA and silica-basedEDFA in 1458-1540 nm wavelength region," Electron. Lett., vol. 39,no. 7, pp. 597-599, Apr. 2003.

16- T. Segi, T. Aizawa, T. Sakai, and A. Wada, "Silica-based composite fiber amplifier with 14801560 nm seamless gain-band," in Proc. Eur. Conf.Optical Communication (ECOC), Amsterdam, The Netherlands, 2001,pp. 228-229.

17- T. Sakamoto, S. Aozasa, M. Yamada, and M. Shimizu, "Hybrid fiber amplifiers consisting of cascaded TDFA and

EDFA for WDM signals," J. Lightw. Technol., vol. 24, no. 6, pp. 2287-2295, Jun. 2006.

18- $\quad$ EKS Bhatia, TS Kamal, RS Kaler Peak-to-average power ratio reduction using coded signal in optical-orthogonal frequency division multiplexing systems IET Optoelectronics 6 (5), 250-254

\section{Figures}

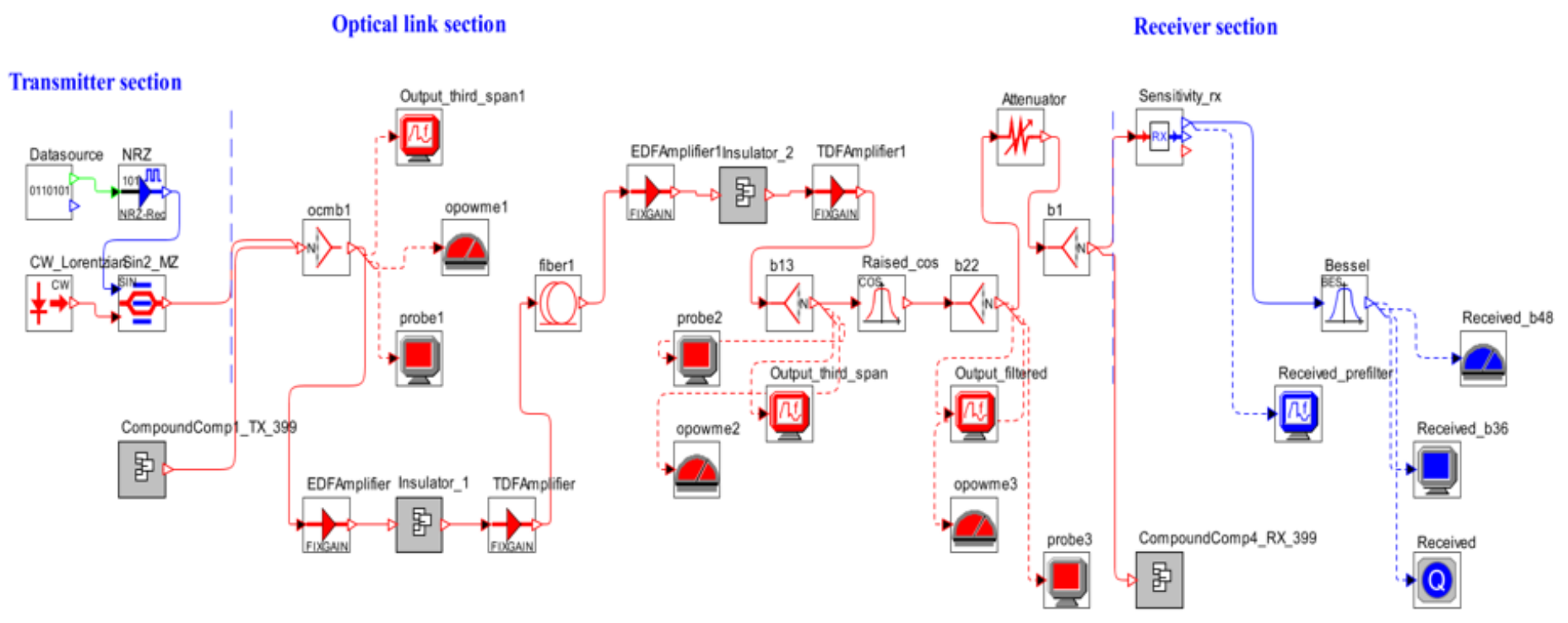

Figure 1

Simulation setup for EDFA and TDFA hybrid optical amplifier 


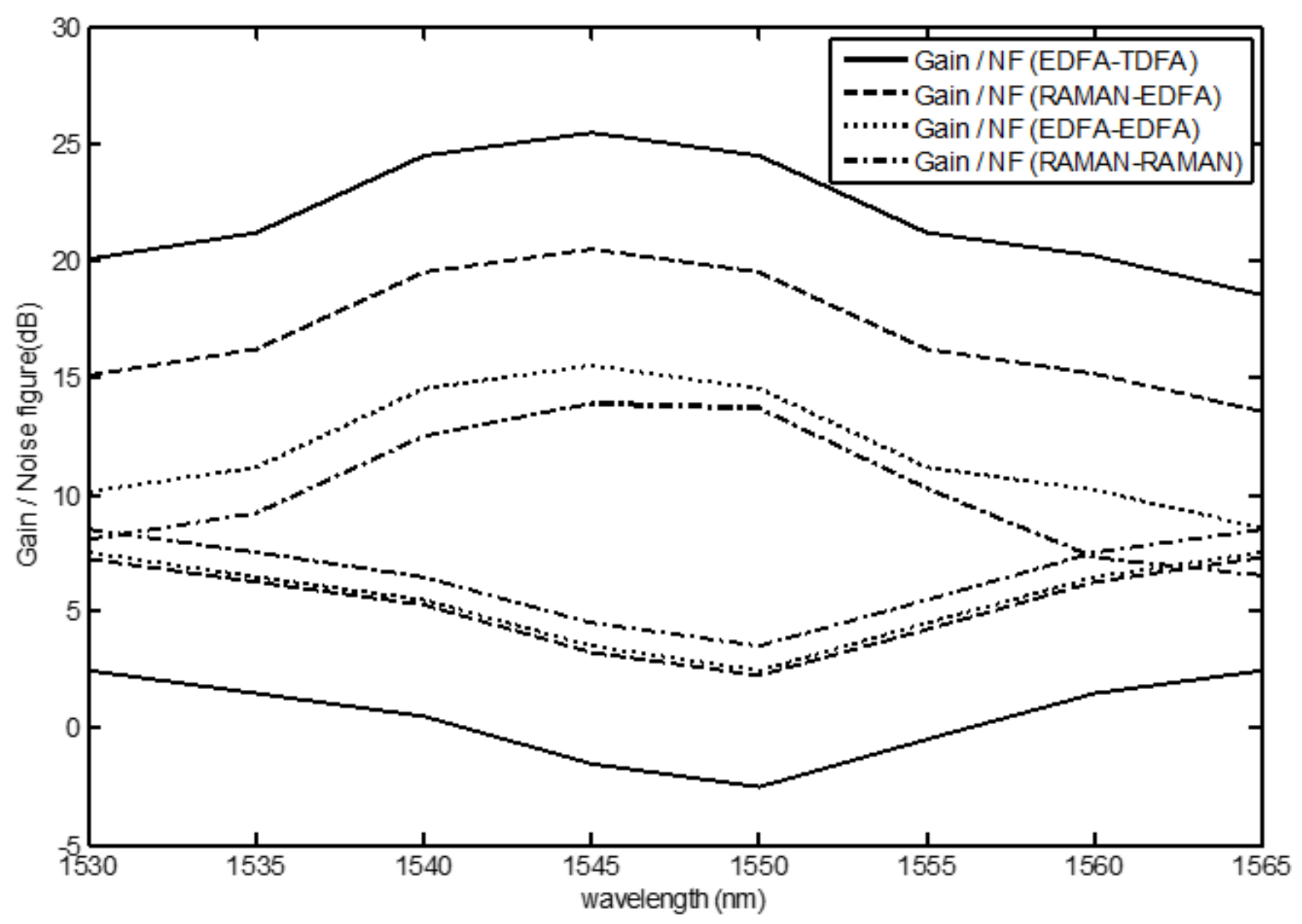

Figure 2

Outcome in terms of gain and noise figure with respect to wavelength 


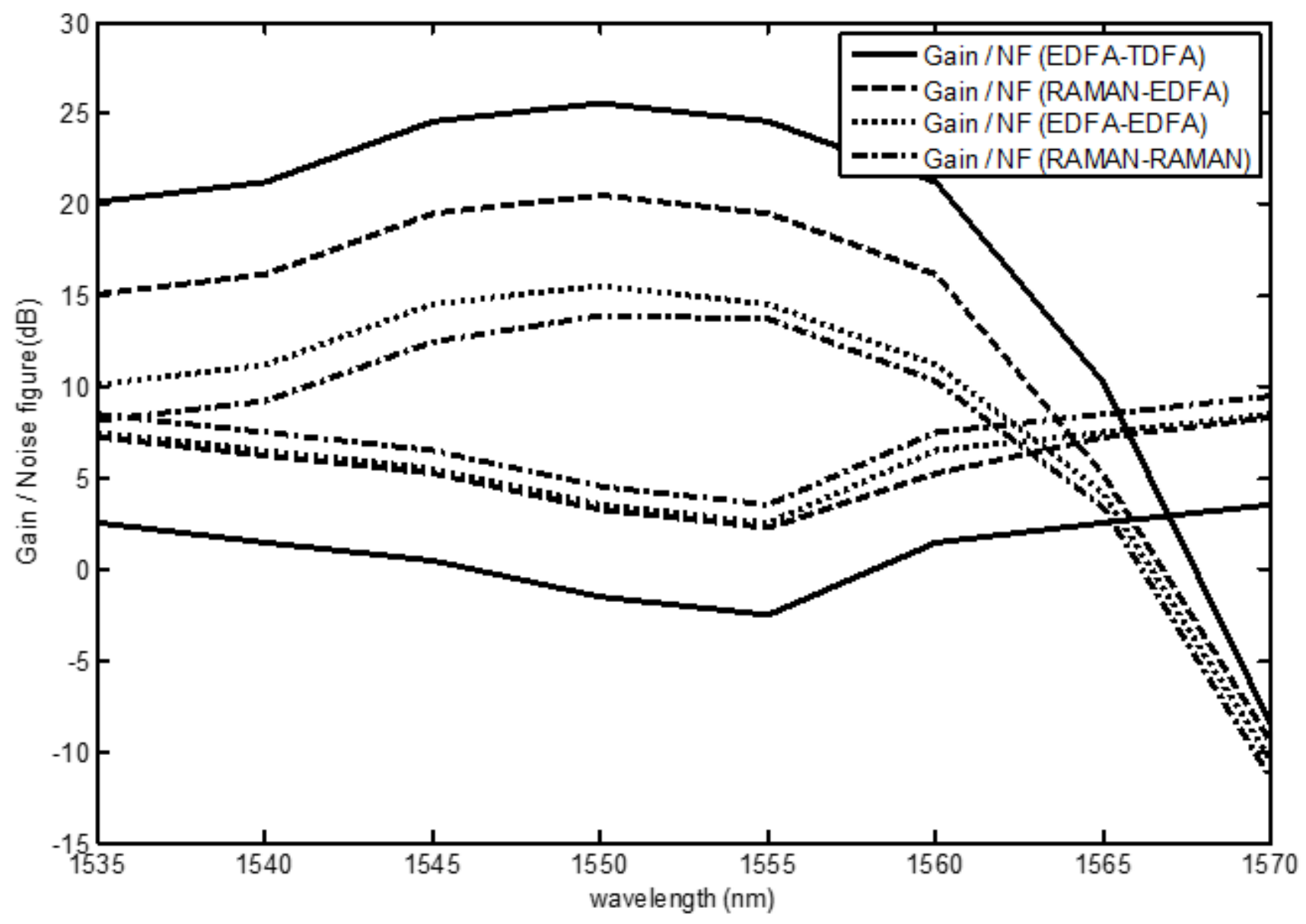

Figure 3

Impact of displacement on channel wavelength on proposed hybrid optical amplifier for gain and noise figure. 


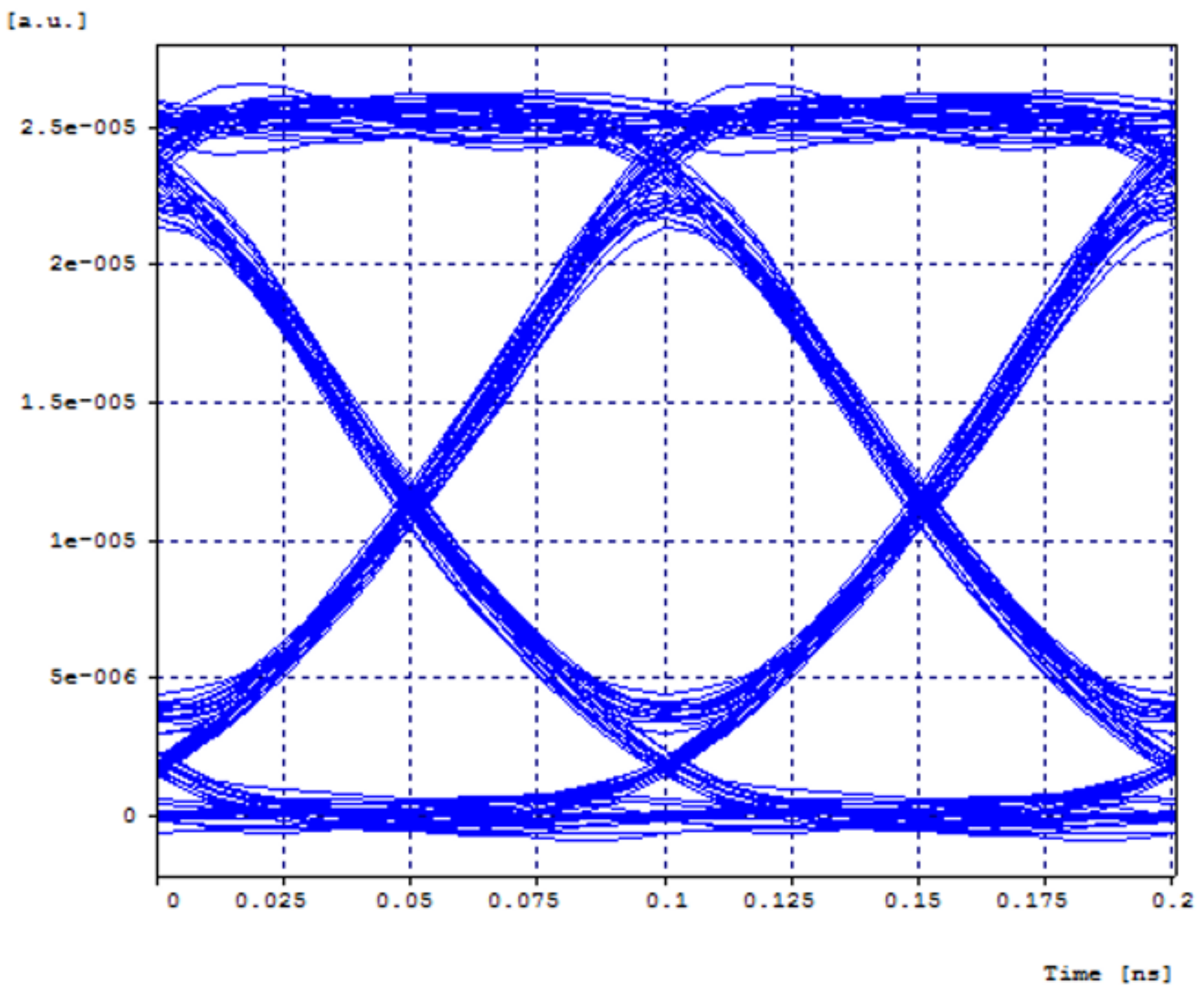

Figure 4

Eye diagram of EDFA-TDFA hybrid optical amplifier 


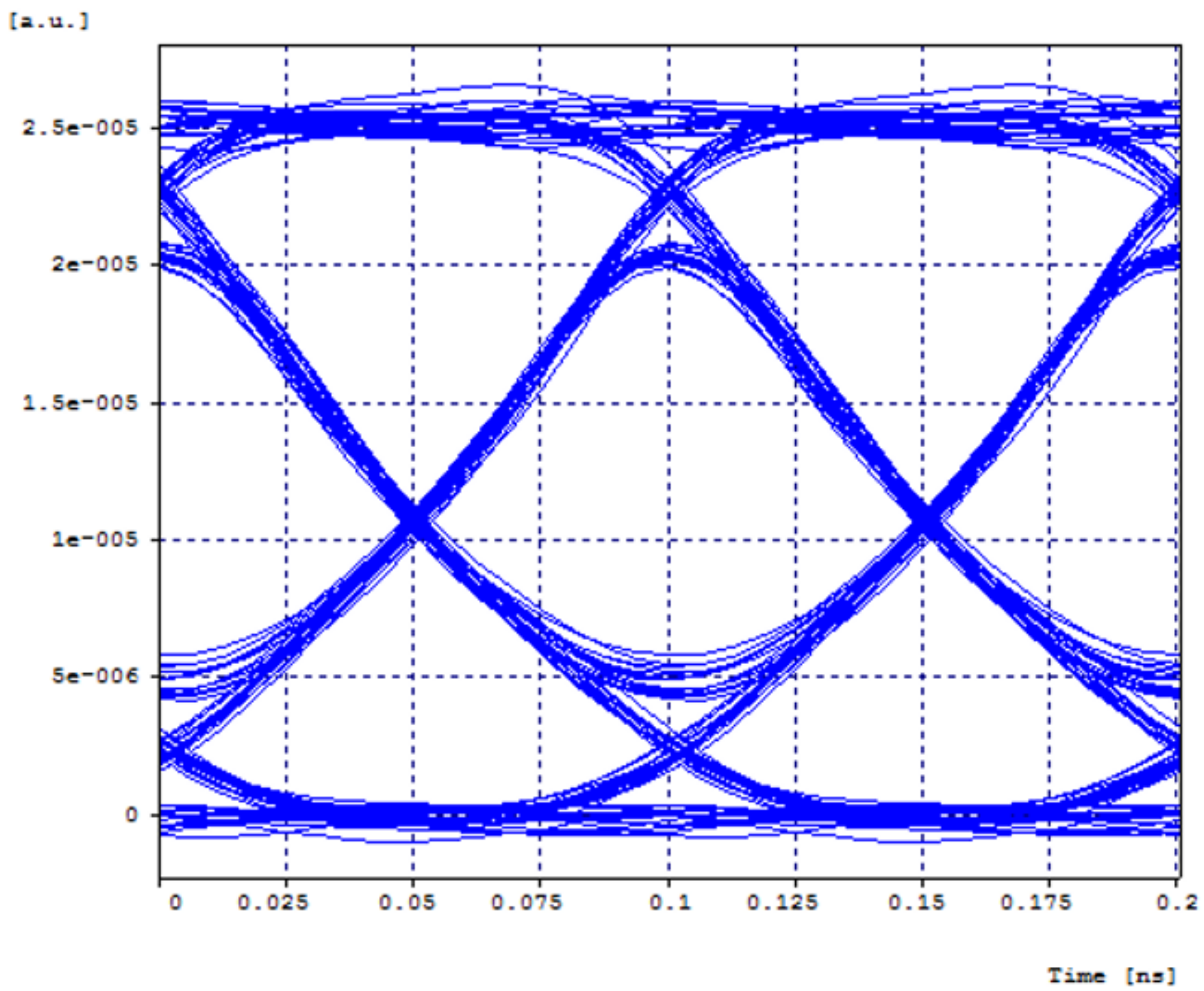

Figure 5

Eye diagram of RAMAN-EDFA hybrid optical amplifier 


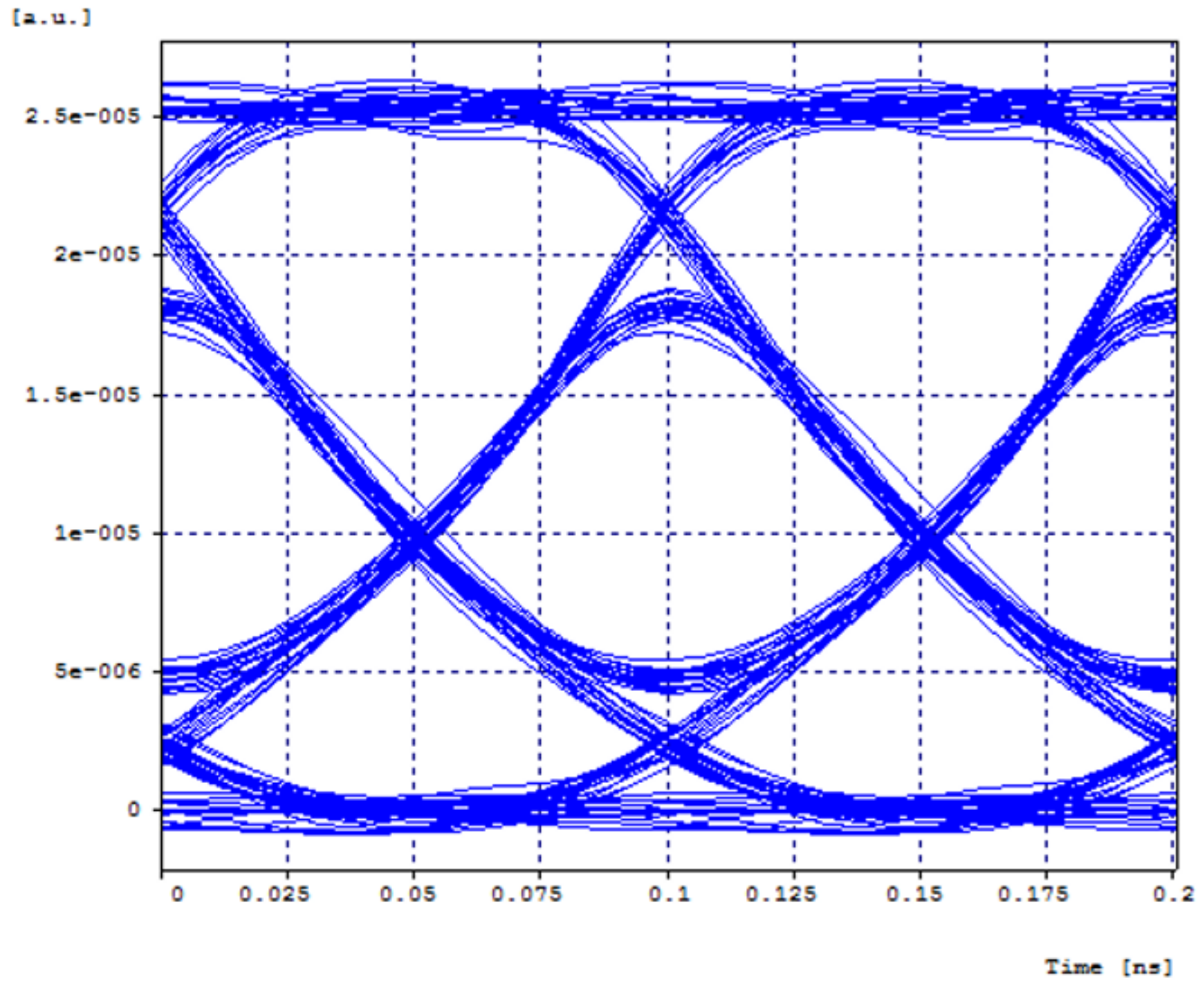

Figure 6

Eye diagram of EDFA-EDFA hybrid optical amplifier 


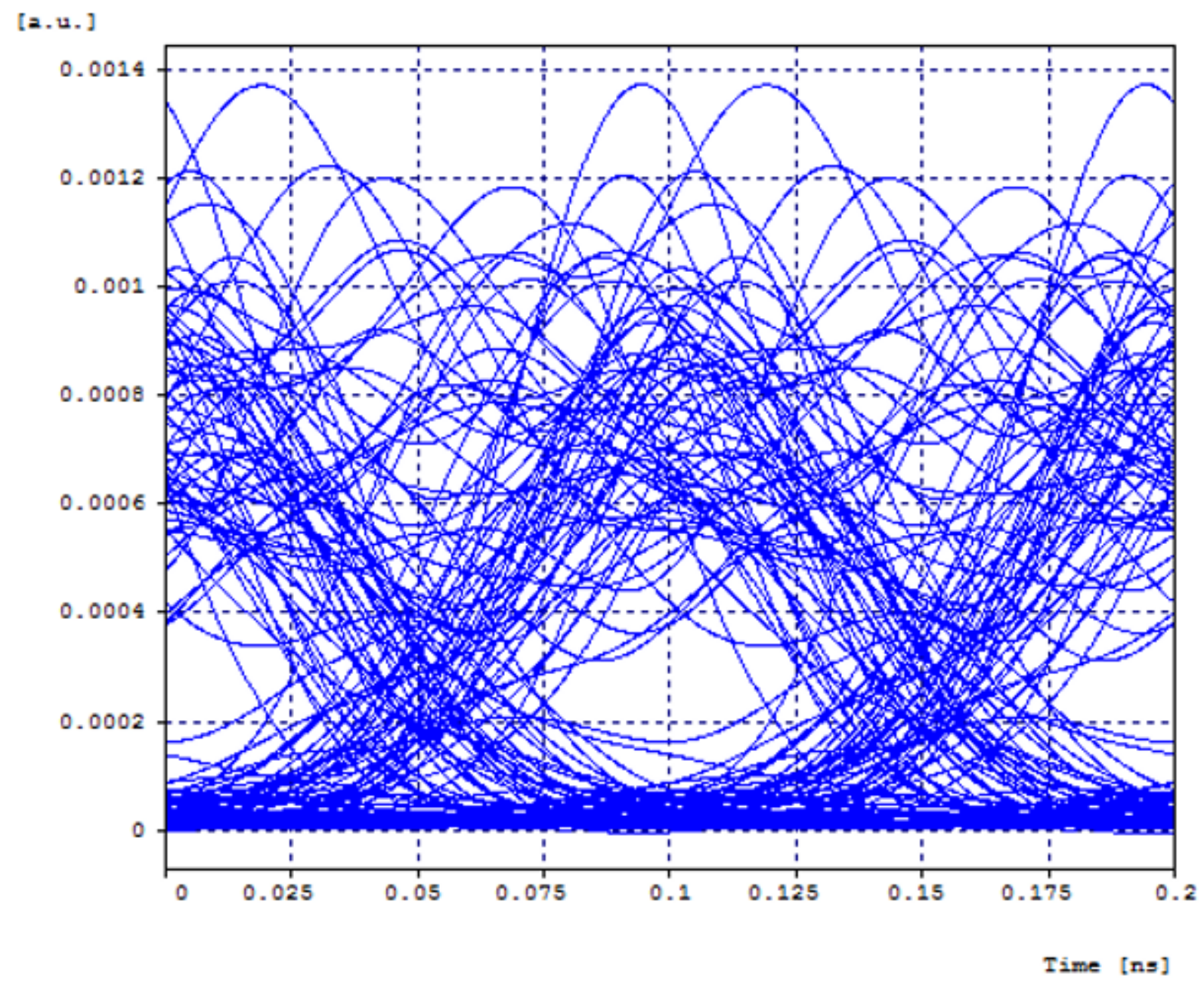

Figure 7

Eye diagram of RAMAN-RAMAN hybrid optical amplifier 


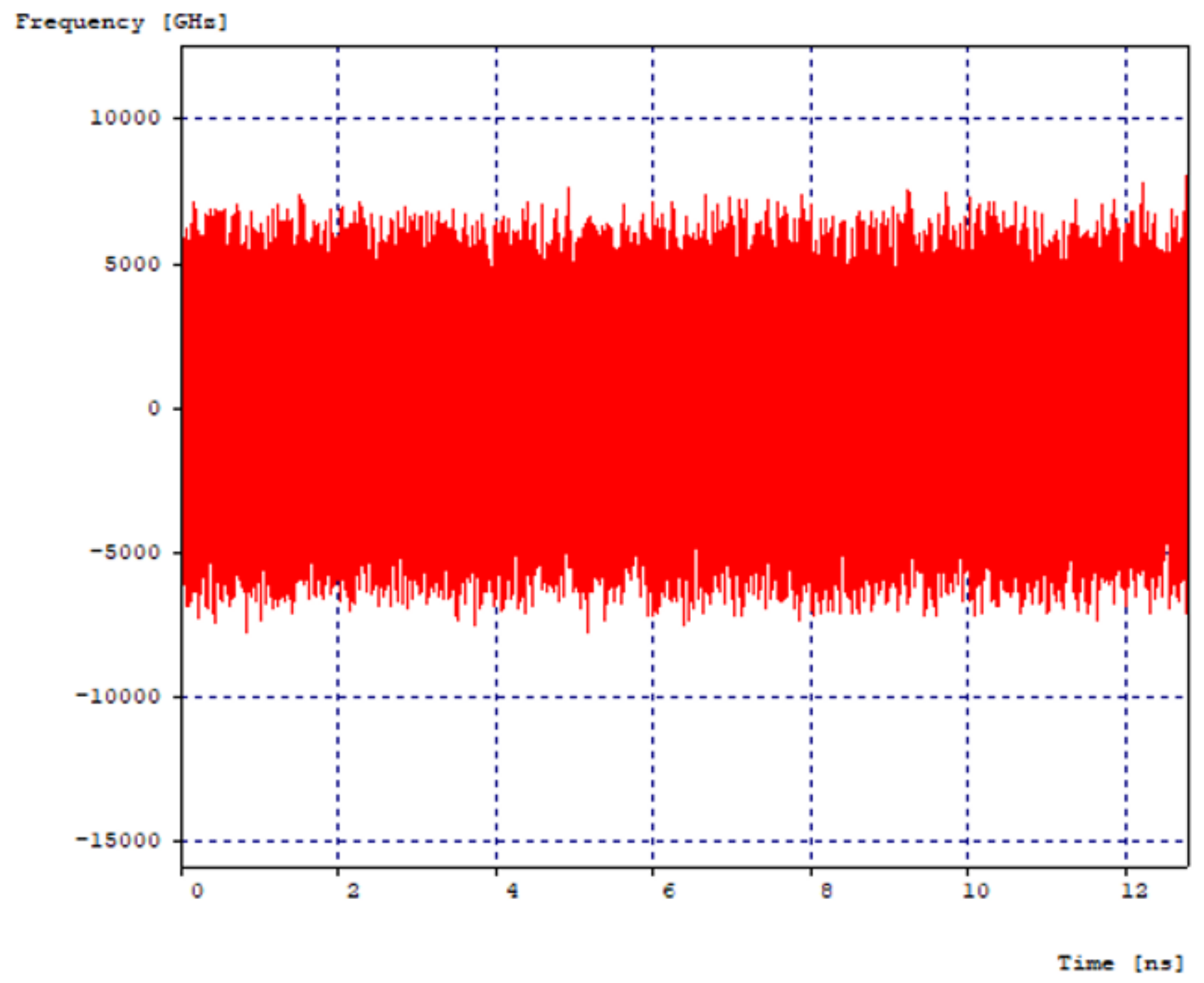

Figure 8

Power amplification in the optical medium from EDFA-TDFA hybrid optical amplifier 


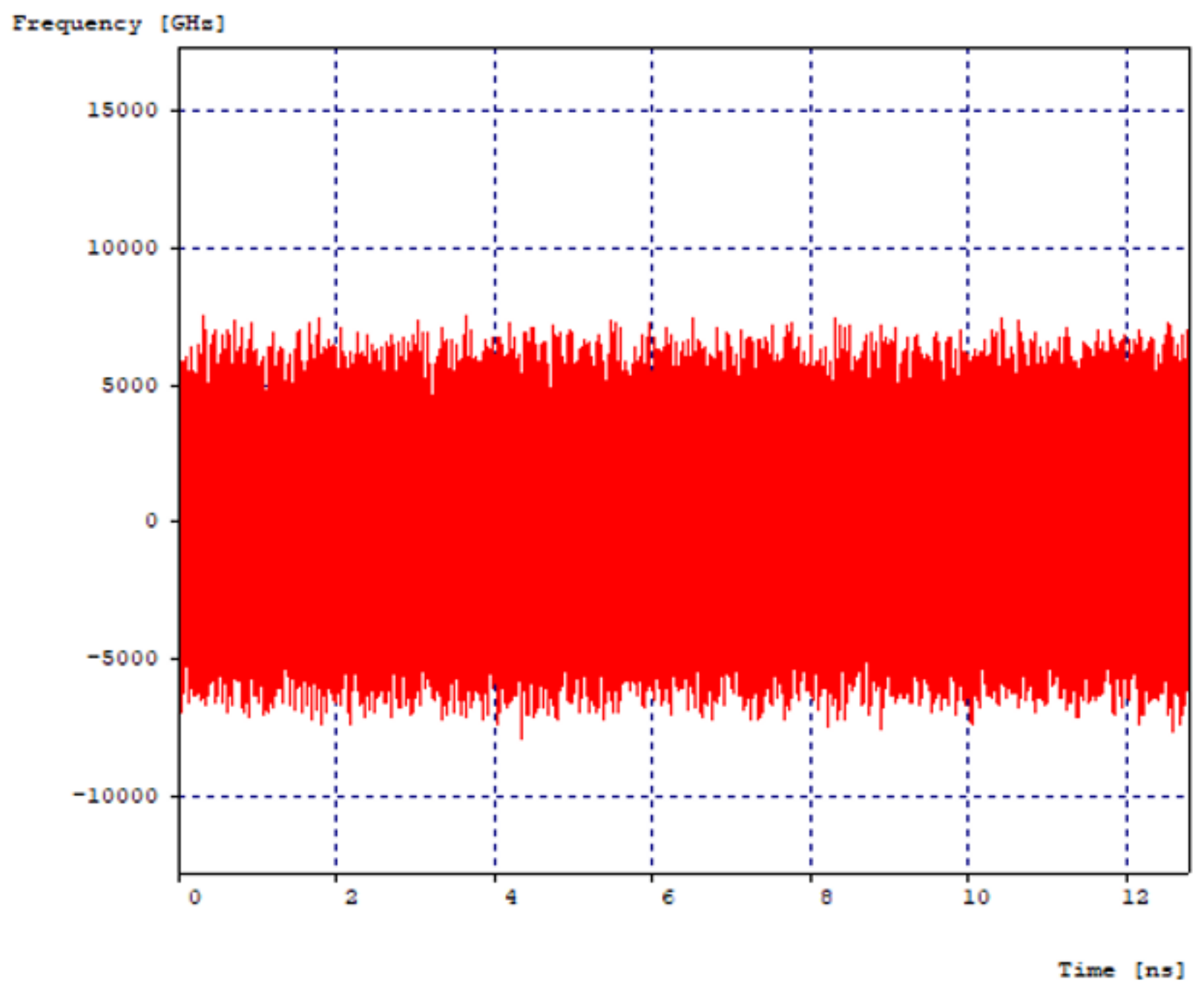

Figure 9

Observation of power amplification near the receiver section from EDFA-TDFA hybrid optical amplifier 
[2. น. ]

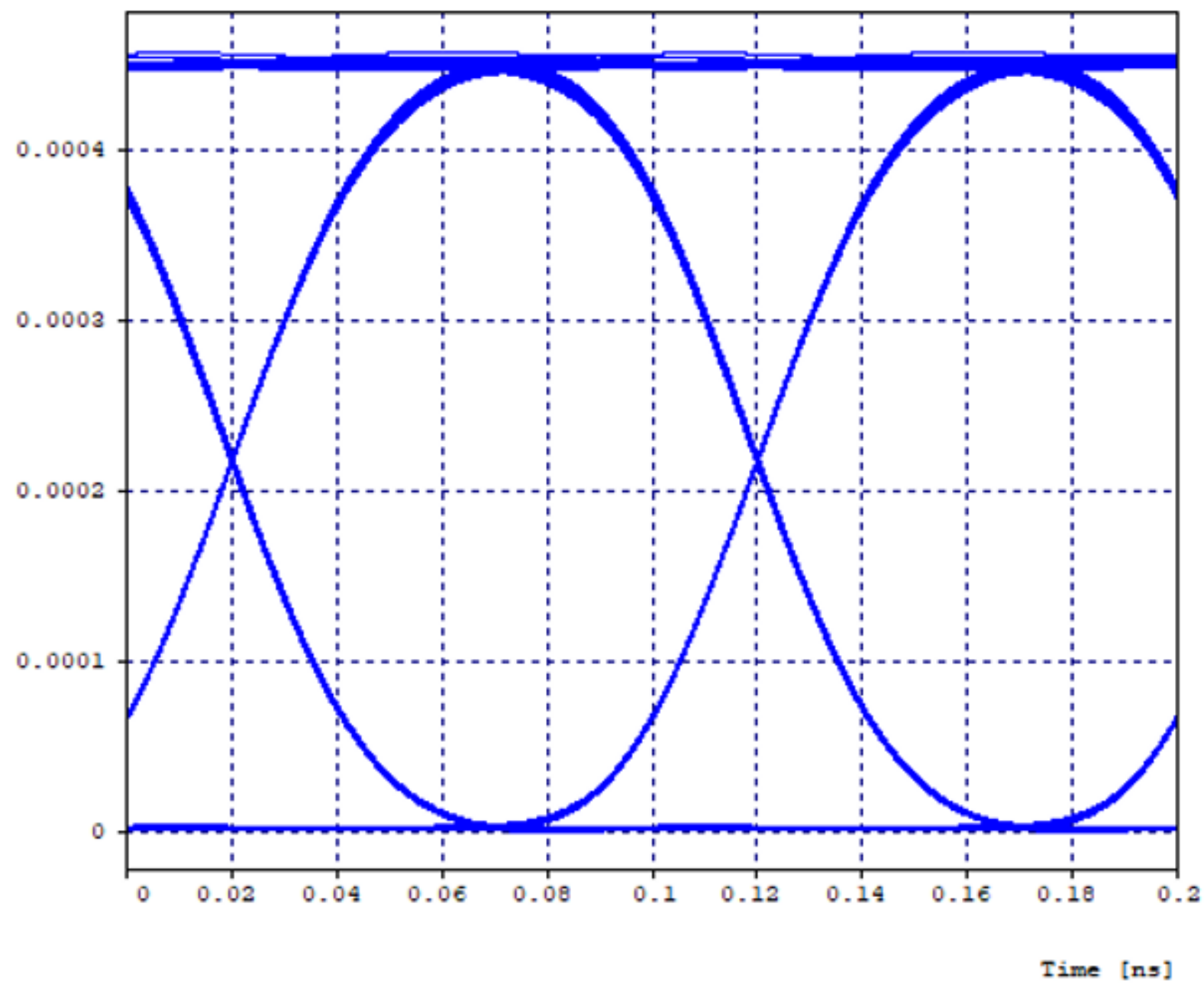

Figure 10

Observation of eye diagram from EDFA-TDFA hybrid optical amplifier in the middle of transmission medium 